\title{
Indoor Navigation in Stadium using Virtual Reality
}

\author{
Swaleha Khan ${ }^{1}$, Ashwini Patil ${ }^{2}$, Gauri Kadam ${ }^{3}$, and Dr.Ashish Jadhav, ${ }^{4} *$ \\ ${ }^{1}$ Ramrao Adik Institute of Technology, Nerul, Navi Mumbai \\ ${ }^{2}$ Ramrao Adik Institute of Technology, Nerul, Navi Mumbai \\ ${ }^{3}$ Ramrao Adik Institute of Technology, Nerul, Navi Mumbai \\ ${ }^{4}$ Ramrao Adik Institute of Technology, Nerul, Navi Mumbai
}

\begin{abstract}
Virtual Reality is a computer generated scenario that simulates a realistic experience. Current popular online maps majorly provide two-dimensional upper views of buildings. It becomes difficult to visualize the interiors of a place. Indoor navigation using VR technology addresses the problem of visualizing the interior infrastructure of huge complex buildings like stadium. This paper 'Indoor Navigation in Stadium using Virtual Reality' aims to provide highly realistic and immersive experience by incorporating an app that uses 360 degree images to serve the purpose. The paper explains how WebVR technology can be used effectively in order to provide a three dimensional view of the stadium with navigation functionalities to the users.
\end{abstract}

\section{Introduction}

Navigation systems have been extensively used in outdoor environments, but indoor navigation systems are still in initial development stages. Modern navigation systems use electronic devices to determine user's current location, find the best possible routes, and in some cases also independently guide users to the destination. Presently, most navigation systems use signals from Global Positioning System (GPS), which works in outdoor environments but has difficulty indoors due to reduced signal strength. Complementary technologies such as Wi-Fi-based and codebased methods have been proposed for indoor navigation; though, a definite solution for the industry has not been established. As the popularity of mobile devices and location-aware applications has increased, indoor navigation systems have become highly beneficial for both personal use and applications in many industries such as retail, entertainment, healthcare, and manufacturing. Some indoor navigation systems provide 360-degree panoramic photos of a location, but do not provide an inside view of some huge complex buildings. Thus, it becomes difficult to visualize the interiors of a place.

Virtual reality technology can be efficiently used in indoor navigation systems. One of the technique of virtual reality is Window on World (WoW), also known as "Desktop VR" or "WebVR", which provides the threedimensional view of world to users through the 'window' of the computer monitor and navigates users through the space with control devices such as mouse or keyboard. The computer monitor screen is used to display virtual reality applications and provides graphical interface to users. Additionally, this concept can be extended to Smartphone or tablet screens too.

*e-mail: ashish.jadhav@ rait.ac.in
However, WebVR apps are not graphic intensive. The complexity of the 3D scene has to be planned carefully. Building an application that is technically as well as socially robust is a lot of work. Sensible planning of the application such that it supports a bigger number of devices is required.

Virtual reality-based solutions can be productively used in entertainment industry as Virtual reality completely engages the user into simulated and interactive environment to provide more enhanced experience. Fans in massive Entertainment Complex like stadium want to be entertained in ways outside of the event itself and in a streamlined, engaging manner. The focus has shifted from a purely "game-focused" experience to more of a "well Enhanced" experience. One of the core expectations from the modern fan is an exciting atmosphere within the Entertainment Complexes. The Virtual Reality centered solution presented in this paper provides indoor navigation facility to enhance fan's experience.

\section{Related work}

The paper on the Introduction to Virtual Reality and its Challenges [1] explains what VR is and its principles. It states how VR is used to simulate real world experiences for its users to get an immersive experience and how the technology gives enormous scope to explore the world of $3 \mathrm{D}$ and your imagination. Its applications spread wide from product development to entertainment. However, its challenges include creating a convincing $3 \mathrm{D}$ virtual environment - the more realistic the environment, the longer it takes to make it. Thus, it gets tedious too. Many systems rely on hardware that encumbers a user or limits his options through physical tethers. 
The paper on Hyper-navigation [2] shows how agents can be used to guide the user in virtual world about actions they can take in their virtual environment, thus helping them escape the virtual world. The interior of buildings and rooms within it are demonstrated for navigation. This is done with the help of task-oriented agents. Another paper referred for navigation [3] proposes view-based navigation system for performing indoor navigation. The navigation to a destination is provided with the help of a single monocular camera wherein having of a smartphone with a good quality camera is a necessity. Thus, it has been observed that indoor navigation can be provided with different ways which can prove beneficial to a number of users.

Ibrahim Arda Cankaya et al. proposed the paper [4] that uses Augmented Reality for the navigation system. This paper introduces the pedometer algorithm that first filters the data according to the axis and also considers the variations that come forth according to the gender. This is done by utilizing smart phone's accelerometer as the pedometer, compass and camera features with augmented reality. Another paper titled Combination of Real and Virtual World for Indoor Navigation using Mobile Application [5] also uses Augmented Reality for performing navigation However, the approach is different. It uses ARCoreSDK that has an inbuilt property called Area Learning which is similar to machine learning that helps to learn the present scenario features which in turn facilitate indoor navigation. Thus, it has been observed that using of Augmented Reality imposes the use of additional hardware or software requirements. Using of Virtual Reality technology cuts down the need of such additional requirements. DesktopVR thus facilitates the combination of Virtual Reality with web development. The JavaScript enabled web browser can be used for virtual navigation.

Quan Ye et al. proposed the paper [6] that talks about VR Interactive Feature of HTML5-based WebVR Control Laboratory by Using Head-mounted Display. It uses Three.js graphic engine based on Web Graphics Library (WebGL) to implement the feature. In order to illustrate the operational approach, a ball and beam system is used. It also explains the concept of 3D modeling, data communication that takes place in virtual reality and lastly a case study on the NCSLab and how they enhanced the experience of the online laboratory using virtual reality. Another paper titled 'Virtual Reality Websites' [7] explains the current SaaS technologies used for Virtual Reality (VR). Thus these papers prove helpful in understanding how VR can be implemented by using WebVR.

QR code technique can be advantageous in navigating the path from current source. In [8], each geographical area is assigned rooms for efficient working of the algorithm implemented. It consists of partition, functional and connectivity number attached to it for precise routing to desired destination. Similarly, another paper [9] used the concept of QR codes to help blind people navigate within huge buildings. Thus, an inference is that QR codes can be used to store location details of a particular place such as the QR code on stadium tickets can be used to retrieve the seat location of the user and help him navigate accordingly.

\section{Proposed Methodology}

\subsection{Problem Definition}

Most maps do provide a navigation system but what is lacking is the detailed indoor navigation for a particular place. The navigation can be provided with the help of location of the person. However, this might fail when the person enters huge buildings or areas where tracking of the location becomes difficult. Also, the floor level and rooms inside the building couldn't be tracked with the help of GPS. Indoor navigation gives an immersive experience to the user with the help of images. These images are enhanced 360 degree images which give better viewer experience than $2 \mathrm{D}$ images. The 360 degree images provide a 360 view from any location captured. Therefore, to guide or navigate visitors in the stadium to their seat they will be given a virtual tour of the stadium by designing a 360 degree interior map of the stadium.

\subsection{System Architecture}

The system is a web application which is divided into 3 major parts: The Front-End, The Middleware and The Back-End. The user interacts with the front-end, which sends requests to the back-end and gets back some response. This is how a typical web VR application works.

Figure 1 below shows what the architecture consists of. The user may or may not have a ticket with a QR code. The user then access the webapp which consists of a MapsServer with the database. The input from the user is assessed and data is retrieved after which the business logic is applied considering the input. The renderer is then used to render the final map back to the user's web browser.

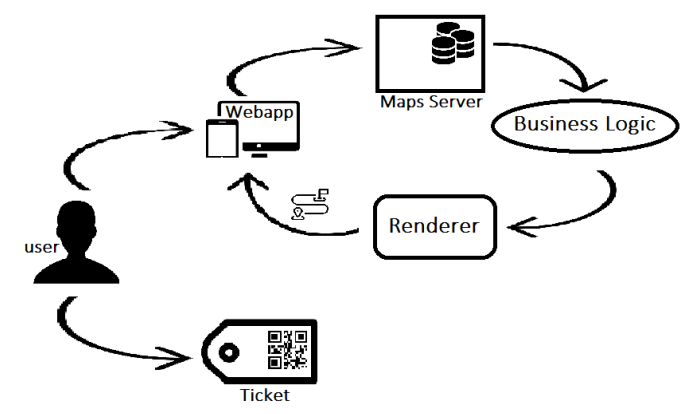

Figure 1. System Architecture.

The Front-End framework used is Angular 4 which is a JavaScript framework along with PhotoSphereViewerJS for rendering the panoramic photo. It sends HTTP Requests to the middleware. Node.js is an application server that serves as the middleware here whereas MongoDB is the NoSQL database daemon process that serves the BackEnd. With the help of Node.js, the requests are being sent to the database to which the database responds accordingly. The images along with marker data are then loaded to the frontend. 


\subsection{System Design}

The core of the system is built using MEAN stack. Users can find the optional route by providing their current location and destination. The 360 degree panoramas are viewed inside PhotoSphereViewer which can be panned or zoomed in any direction. The map of the stadium is represented as a Graph structure where pictures are nodes and the edges represent adjacency. The system then calculates the minimum hops to load an image with the help of Adjacency matrix and accordingly provides virtual directions to reach from source to destination.

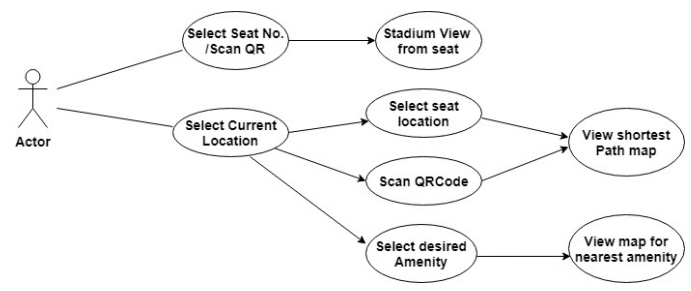

Figure 2. Use Case Diagram.

Use Case Diagram in Figure 2 shows in detail the varied options available for the user to perform as the webapp loads as well as the expected outputs received depending on the input.

Upon initialization of web application, the user has following options:

- The user can also search for a specific location with the help of a search box provided.

- Enter any gate number as source and then scan the QR code which recognizes the seat number or enter the destination manually.

- Autoplay the desired path from the source or use markers that will have tooltip which will hint the user about the next path that will be loaded.

- Get the path from any particular location to any stadium amenity nearest to him.

Data flow diagram in Figure 3 describes the data flow of proposed system in detail wherein four inputs are taken from the user and accordingly the backend processes the request. The output received from the system helps navigate the user to the destination.

\subsection{Implementation}

\section{- Capturing panoramic photos:}

360 degree photographs of each node are captured for each node. The camera used for this purpose is Samsung gear 360. ActionDirector is used to get a complete photo sphere.

- Storing panorama data and marker: data into database:

Every node's data is stored in MongoDB which is NoSQL database. The markers present in each photo sphere with Latitude and Longitude of the marker and tooltip data are stored in the database.

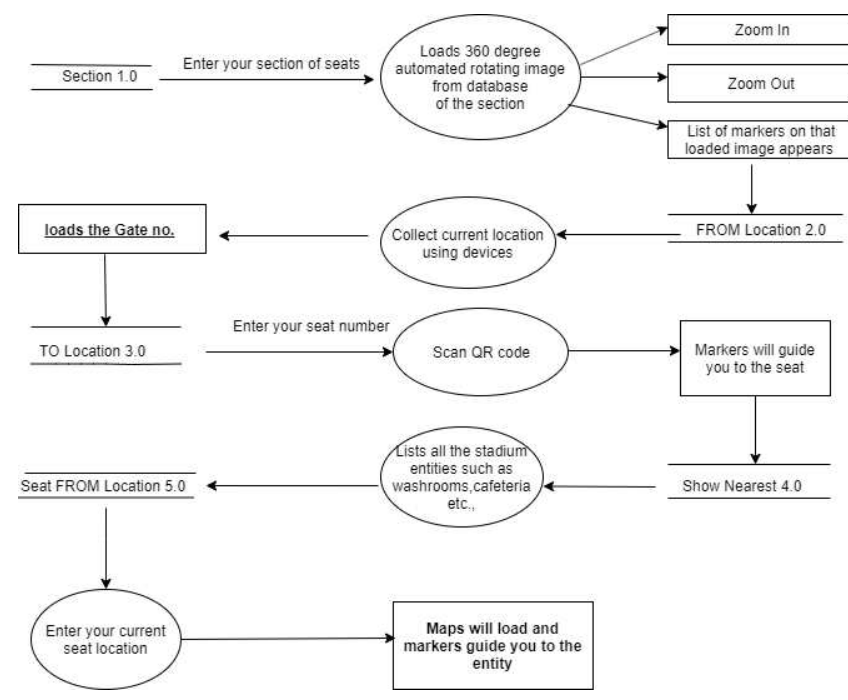

Figure 3. Data Flow Diagram.

\section{- Creating graph data structure:}

There is a need to find the shortest path between two nodes for which a JS script is used for creating a graph data structure and making inter-connections between nodes.

\section{- Developing web Application.}

The system is a web application which the user can access using any modern JavaScript enabled web browser.

\section{Results and Discussions}

Figure 4 shows the first page that loads when you open the Webapp.

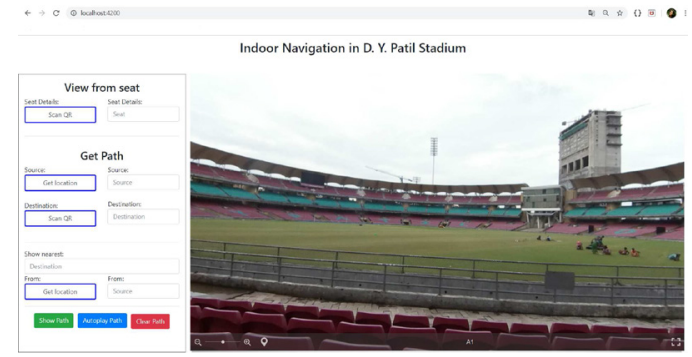

Figure 4. System User Interface.

As explained through Figure 2, the closer look to the interface shown in Figure 5 shows the varied options available to the user for navigating in the stadium.

The 'View from seat' section lets the user get an entire 360 degree view from any particular seat and helps the user to decide during seat booking. The 'Get Path' section is the one which helps navigate by providing a map to the user along with markers. The source and destination for navigation to a particular seat can either be manually entered or user's location can be detected for getting 


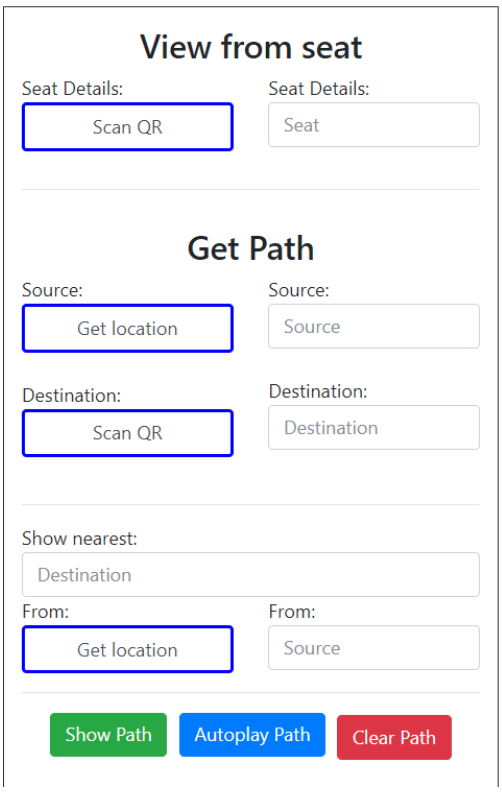

Figure 5. Options available.

source information and QR on the ticket can be scanned for seat information. The subsection of Get Path is to show path for the nearest desired amenity from a source that can again be detected from user's location or be manually entered.

The three buttons at the bottom are submit buttons that send request to the server. The Show Path renders the map to the user along with markers that help users reach the destination. The Autoplay Path too renders the map but autoplays the entire path to the user without having the user to click on markers. The Clear Path button clears the fields entered above and refreshes back to the start page of the webapp.

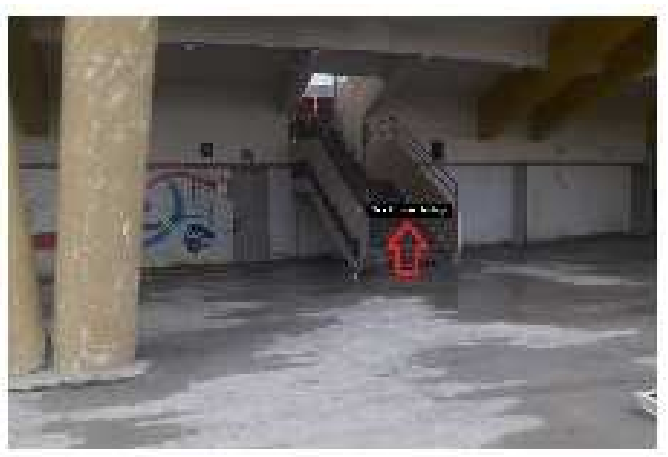

Figure 6. Marker

Figure 6 shows how the marker appears on an image along with the tooltip information on the marker. The system loads the first image with markers. Further, if a marker is clicked upon, it will load the next image that is linked to that particular marker. This process can continue even if the fields on the left are not entered. Also, this way the system can support large number of users since every mobile phone is running their own instance of the webapp locally and accordingly the map is rendered.

\begin{tabular}{|c|c|c|}
\hline Parameter & 2D image & $\begin{array}{c}\text { 360 degree } \\
\text { image }\end{array}$ \\
\hline Total number of images for the stadium & $360-400$ & $90-100$ \\
\hline Approximate size of each image & $50-80 \mathrm{~KB}$ & $8-10 \mathrm{MB}$ \\
\hline Load on database wrt size of image & Low & High \\
\hline Load on database wrt number of images & High & Low \\
\hline Markers per image & $l$ & $2-4$ \\
\hline $\begin{array}{c}\text { Response time for navigation in the map rendered } \\
\text { on the app }\end{array}$ & Fast & Fast \\
\hline $\begin{array}{c}\text { Worst case number of images to be downloaded } \\
\text { to the app for navigation }\end{array}$ & 30 & 20 \\
\hline $\begin{array}{c}\text { Best case number of images to be downloaded to } \\
\text { the app for navigation }\end{array}$ & 4 & 1 \\
\hline User Experience rating according to survey taken & 3 & 9 \\
\hline
\end{tabular}

Figure 7. 2D vs 360

The webapp includes 360 degree images instead of simple 2D images. A con of using the 360 degree images is the image size since 360 degree images are large in size. However, there are many pros with respect to the user experience. Markers can be embedded on 2D images as well but the number of images will increase with 2D images since a 2D image cannot show every route or turn possible from any particular position the user is at. Thus, even though the size of images is reduced with 2D images, the number of images increase. This has been explained in Figure 7 through a table which also shows the rating for the user experience. The survey was conducted among the students of RAIT, Nerul wherein the average score on 10 is calculated and shown in the table. The numbers given in the table are approximate.

The traditional alternative to indoor navigation is by following the sign boards in the stadium or by asking other individuals present in there. However, both are inconvenient since they waste time of the user. With a VR-based Indoor Navigation, the user can not only plan their day beforehand and utilise time for a better purpose but also get an immersive experience of the interiors of a stadium. The manpower required for guiding the spectators also reduces.

The system runs in its worst case when the source is at one end of the stadium and the destination is at the other opposite end. In this case, quite a lot number of markers have to be traversed and clicked upon to reach to the destination which at times may seem cumbersome to the user. The system runs in its best case when the destination is adjacent to the source with respect to the adjacency matrix.

\section{Conclusion}

This paper proposes an indoor navigation for stadium using The WebVR technology providing the user with a complete riveting experience. The feature of $\mathrm{QR}$ code scanning on the ticket brings an effortless solution to the user thus increasing feasibility. The virtual shortest path to the destination is shown with the help of 360 degree images. This is achieved by calculating adjacency matrix between the images. The proposed system works even on simple low end devices like mobile phones or even a low 
end desktop PC. Virtual reality is traditionally associated with high end computing for which a wide range of hardware and software is being used. However, a serious concern about this technology is related to the issue of privacy. A stranger knowing so many things about your indoor infrastructure might become a security issue. The security issues need to be addressed before implementing applications that involve covering the secured infrastructure.

\section{Acknowledgment}

We thank The D.Y. Group for the constant cooperation by giving us the permission to click images and to access the infrastructure of The D. Y. Patil Stadium as and when required for the project.

\section{References}

[1] Sharmistha Mandal, Brief Introduction of Virtual Reality and its Challenges 4, (2013)

[2] J. Emhardt , J. Semmler and T. Strothotte, Hypernavigation in virtual buildings $\mathbf{6},(2002)$
[3] Mitsuaki Nozawa , Yoshinobu Hagiwara and Yongwoon Choi, Indoor human navigation system on smartphones using view-based navigation, 1916-1919 (2012)

[4] Ibrahim Arda Cankaya, Arif Koyun, Tuncay Yigit and Asim Sinan Yuksel, Mobile Indoor Navigation System in iOS Platform Using Augmented Reality, (2015)

[5] Vidhyavani.A, StephinStanly, Ankit Kumar Pandey and Shivam Choudhury, Combination Of Real And Virtual World For Indoor Navigation using Mobile Application 8, (2019)

[6] Quan Ye, Wenshan Hu, Hong Zhou, Zhongcheng Lei, Shiqi Guan, VR Interactive Feature of HTML5-based WebVR Control Laboratory by Using Head-mounted Display 14, (2018)

[7] Vinit Sathe , Piyush Gupta , Karan Kaushik , Suvarna Bhat and Sachin Deshpande, Virtual reality websites(VR WEB) 1, 647-652 (2017)

[8] Ying Zhuang, Yuhao Kang, Lina Huang and Zhixiang Fang, A Geocoding Framework for Indoor Navigation based on the QR Code, 1-4 (2018)

[9] Affan Idrees, Zahid Iqbal and Maria Ishfaq, An efficient indoor navigation technique to find optimal route for blinds using QR codes, 690-695 (2015) 